EPJ Web of Conferences 40, 06008 (2013)

DOI: $10.1051 /$ epjconf/20134006008

(C) Owned by the authors, published by EDP Sciences, 2013

\title{
Hard magnetic properties of melt-spun Mn-Al-C alloys
}

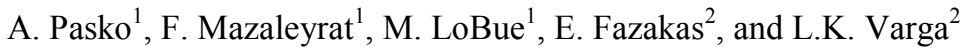 \\ ${ }^{1}$ SATIE, ENS Cachan, CNRS, UniverSud, 61 av President Wilson, F-94235 Cachan, France \\ ${ }^{2}$ Wigner RCP, HAS, ISSPO, H-1525 Budapest, POB 49, Hungary
}

\begin{abstract}
Structural and magnetic characterization of $\mathrm{Mn}_{x-y} \mathrm{Al}_{100-x-y} \mathrm{C}_{2 y}(x=\{50,55\} ; y=\{0,1\})$ melt-spun ribbons is reported. To obtain the metastable ferromagnetic $\tau$-phase, rapidly solidified alloys were annealed either in a vacuum furnace at $823 \mathrm{~K}$ or directly in the vibrating sample magnetometer under applied magnetic field. Optimal magnetic properties were demonstrated by $\mathrm{Mn}_{54} \mathrm{Al}_{44} \mathrm{C}_{2}$ samples proved to be single-phase with a coercivity of $0.19 \mathrm{~T}$ measured in both cases. For this composition the structural $\varepsilon \rightarrow \tau$ phase transformation has been magnetically detected at $786 \mathrm{~K}$, Curie temperature of $\tau$-phase $\left(T_{\mathrm{c}}=592 \mathrm{~K}, T_{\mathrm{p}}=610 \mathrm{~K}\right)$ has been determined using mean field approximations in ferromagnetic and paramagnetic regions. Rietveld refinement of X-ray diffraction spectra was employed to analyse the phase constitution of annealed alloys, lattice parameters as a function of chemical composition and mean grain size for the phases involved.
\end{abstract}

\section{Introduction}

$\mathrm{Mn}-\mathrm{Al}$ alloys are attractive candidates for permanent magnet applications due to good magnetic properties (superior to conventional ferrites), high technological properties (mechanical strength, machinability, corrosion resistance) and low cost (no rare earth elements). The ferromagnetic tetragonal $\tau$-phase with strong uniaxial magnetocrystalline anisotropy $[1,2]$ forms from the nonmagnetic hexagonal $\varepsilon$-phase by annealing a quenched alloy with $50-60 \%$ of $\mathrm{Mn}$ at approximately $823 \mathrm{~K}$. Both phases are metastable at room temperature and therefore need special thermal treatment. Carbon addition stabilizes $\tau$-phase and facilitates production of commercial anisotropic materials by hot deformation [3]. Magnetic hysteresis of Mn-Al-C alloys is sensitive to the microstructure and presence of defects developed during $\tau$-phase formation, comprising martensitic or massive transformation mechanisms [4], and is therefore strongly influenced by the production route.

Various production techniques have been employed for preparation of homogeneous Mn-Al-C alloys: meltspinning [5-7], levitation melting with splat quenching [8], spark erosion [9], ball milling [10, 11], mechanical grinding [12]. Depending on the solidification rate or size reduction degree, fine-grained materials with improved functional properties can be obtained. In this paper we report on the structural and magnetic characterization of $\mathrm{Mn}-\mathrm{Al}-\mathrm{C}$ melt-spun ribbons. The goal is to investigate a correlation between the production route, phase constitution and resulting hard magnetic properties. Using accurate X-ray crystallographic analysis and magnetization measurements in a wide temperature range, we have advanced our understanding of structural and magnetic phase transitions in Mn-Al-C system and particular role of carbon.

\section{Experimental}

Binary alloys $\mathrm{Mn}_{50} \mathrm{Al}_{50}$ and $\mathrm{Mn}_{55} \mathrm{Al}_{45}$, as well as their ternary counterparts doped with carbon $\mathrm{Mn}_{49} \mathrm{Al}_{49} \mathrm{C}_{2}$ and $\mathrm{Mn}_{54} \mathrm{Al}_{44} \mathrm{C}_{2}$ were selected for the present study. The ingots prepared by induction melting were cast into $\sim 3 \mathrm{~mm}$ wide and $\sim 25 \mu \mathrm{m}$ thick ribbons using a meltspinning technique. The details of sample preparation can be found in [6]. Final annealing was done in a vacuum furnace at $823 \mathrm{~K}$ for $10 \mathrm{~min}$ with ramping rate $10 \mathrm{~K} / \mathrm{min}$ or directly in the magnetometer cell. Crystal structures were examined by a PANalytical X'Pert Pro X-ray diffractometer (XRD) in $\mathrm{Co}-\mathrm{K}_{\alpha}$ radiation equipped with an X'Celerator linear detector. Rietveld full-profile analysis of XRD spectra was performed using MAUD software [13]. Magnetization curves were recorded on a Lake Shore 7400 Series vibrating sample magnetometer (VSM) equipped with a variable temperature assembly.

\section{Results and discussion}

\subsection{Phase analysis}

The phase composition of rapidly solidified ribbons has been determined by XRD both in the as-quenched state (AQ) and after subsequent heat treatment (HT) as 
described above. According to the phase diagram of AlMn system [14], formation of two equilibrium phases is expected: disordered cubic $\beta$-phase (cP20) with excess of $\mathrm{Mn}$ and ordered trigonal $\gamma_{2}$-phase (hR26) with excess of Al. In addition, two metastable phases may appear in non-equilibrium conditions: disordered hexagonal $\varepsilon$ phase (hP2) and ordered tetragonal $\tau$-phase (tP2). Stable at higher temperatures, $\varepsilon$-phase can be preserved on quenching and transformed into ferromagnetic $\tau$-phase by a composition-invariant (involving only short-range diffusion) structural transition. Depending on the initial chemical composition and thermal history, all these phases were observed in different combinations. Figure 1 presents XRD spectra from the as-quenched samples, while Figure 2 shows them after annealing at $823 \mathrm{~K}$.

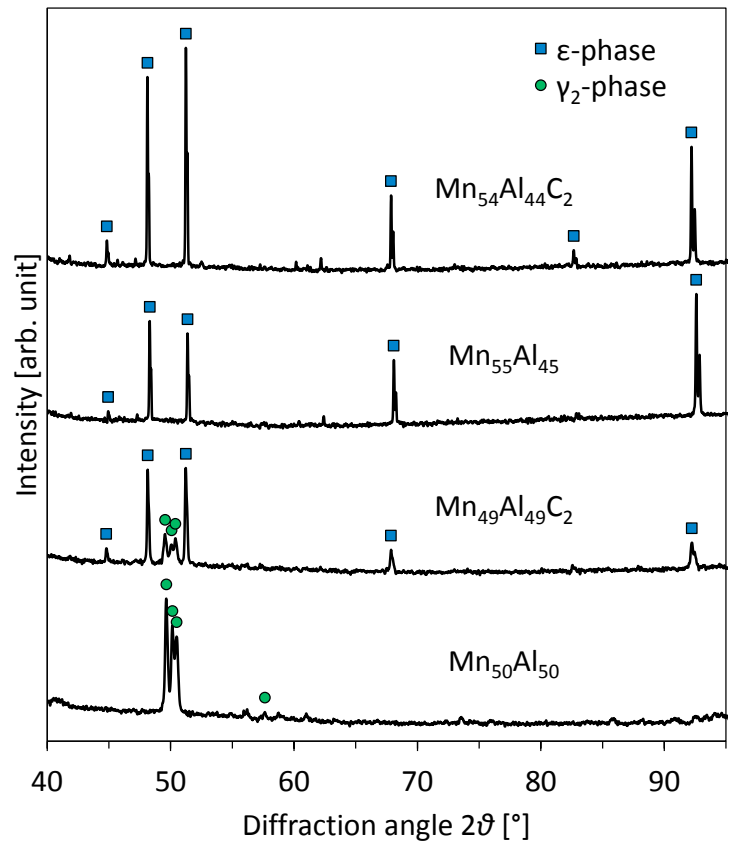

Fig. 1. XRD patterns of the as-quenched samples.

The results of quantitative phase analysis based on Rietveld refinement of XRD data are presented in Table 1. The weight fraction and lattice parameters for each observed crystal structure have been determined. As-quenched equiatomic alloy $\mathrm{Mn}_{50} \mathrm{Al}_{50}$ is composed entirely of $\gamma_{2}$-phase which remains also after annealing. The presence of carbon in $\mathrm{Mn}_{49} \mathrm{Al}_{49} \mathrm{C}_{2}$ helps to retain some amount of $\varepsilon$-phase subsequently transforming to $\tau$ phase. However, $\gamma_{2}$-phase persists and is not substantially affected by heat treatment. On the other hand, Mn-rich composition $\mathrm{Mn}_{55} \mathrm{Al}_{45}$ is more favourable for $\varepsilon$-phase formation. In this case, however, $\beta$-phase precipitates along with desirable $\tau$-phase on annealing. Finally, positive influence of carbon allows to obtain a $100 \% \tau$ phase in ternary $\mathrm{Mn}_{54} \mathrm{Al}_{44} \mathrm{C}_{2}$ sample.

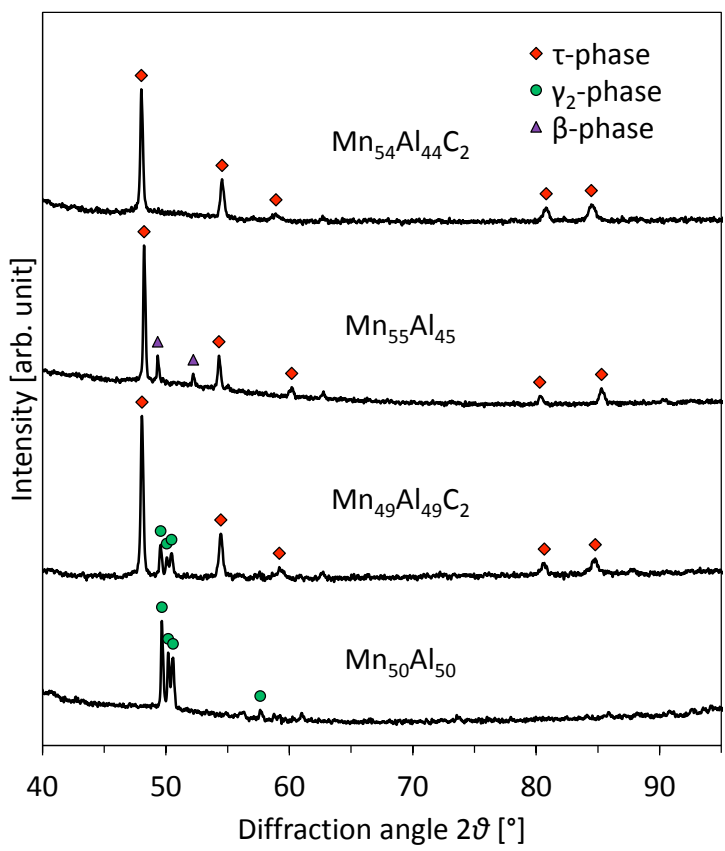

Fig. 2. XRD patterns of the samples annealed at $823 \mathrm{~K}$.

As seen in Table 1, addition of carbon slightly increases both lattice parameters of $\varepsilon$-phase, while in $\tau$ phase it leads to decrease of $a$ and pronounced $(\sim 1.5 \%)$ increase of $c$. The unit cell volume also increases. Though $\mathrm{C}$ has been reported to occupy $(1 / 2,1 / 2,1 / 2)$ sites together with $\mathrm{Al}[15]$, this observation provides a new evidence for presence of carbon atoms in the interstitial positions $(0,0,1 / 2)$ and $(1 / 2,1 / 2,0)$ of tetragonal $\tau$-phase $[10,16]$ similar to Fe-C martensite.

Table 1. Nominal composition of the melt-spun samples and XRD phase analysis (weight fraction, lattice parameters) in the as-quenched state (AQ) and after heat treatment at $823 \mathrm{~K}(\mathrm{HT})$.

\begin{tabular}{|c|c|c|c|c|c|c|c|c|c|c|c|c|c|c|}
\hline \multicolumn{3}{|c|}{ Composition, at. \% } & \multirow[t]{2}{*}{ State } & \multicolumn{3}{|c|}{$\varepsilon$-phase } & \multicolumn{3}{|c|}{$\tau$-phase } & \multicolumn{3}{|c|}{$\gamma_{2}$-phase } & \multicolumn{2}{|c|}{$\beta$-phase } \\
\hline Mn & $\mathrm{Al}$ & $\mathrm{C}$ & & wt. $\%$ & $a, \AA$ & $c, \AA$ & wt. $\%$ & $a, \AA$ & $c, \AA$ & wt. $\%$ & $a, \AA$ & $c, \AA$ & wt. $\%$ & $a, \AA$ \\
\hline \multirow{2}{*}{50} & \multirow{2}{*}{50} & \multirow{2}{*}{ - } & AQ & - & - & - & - & - & - & 100 & 12.59 & 7.898 & - & - \\
\hline & & & HT & - & - & - & - & - & - & 100 & 12.57 & 7.897 & - & - \\
\hline \multirow{2}{*}{49} & \multirow{2}{*}{49} & \multirow{2}{*}{2} & $\mathrm{AQ}$ & 58 & 2.711 & 4.388 & - & - & - & 42 & 12.61 & 7.918 & - & - \\
\hline & & & HT & - & - & - & 70 & 2.767 & 3.619 & 30 & 12.59 & 7.916 & - & - \\
\hline \multirow{2}{*}{55} & \multirow{2}{*}{45} & \multirow{2}{*}{-} & $\mathrm{AQ}$ & 100 & 2.703 & 4.373 & - & - & - & - & - & - & - & - \\
\hline & & & HT & - & - & - & 75 & 2.775 & 3.574 & - & - & - & 25 & 6.437 \\
\hline \multirow{2}{*}{54} & \multirow{2}{*}{44} & \multirow{2}{*}{2} & AQ & 100 & 2.709 & 4.389 & - & - & - & - & - & - & - & - \\
\hline & & & HT & - & - & - & 100 & 2.762 & 3.638 & - & - & - & - & - \\
\hline
\end{tabular}


The mean size of reflecting crystallites determined from line broadening is $100-150 \mathrm{~nm}$ for most phases. Exception is $\varepsilon$-phase having large crystallites of $\sim 600 \mathrm{~nm}$ and a noticeable $\left(\begin{array}{lll}0 & 0 & 1\end{array}\right)$ preferred orientation in asquenched samples which may be attributed to a columnar microstructure. Further refinement of grain size can be achieved by increase of the cooling rate in the meltspinning setup.

\subsection{Magnetic properties}

Magnetic measurements were performed on rapidly solidified ribbons in the as-quenched state, after heat treatment in the furnace, and also during in-situ heating/cooling in VSM.

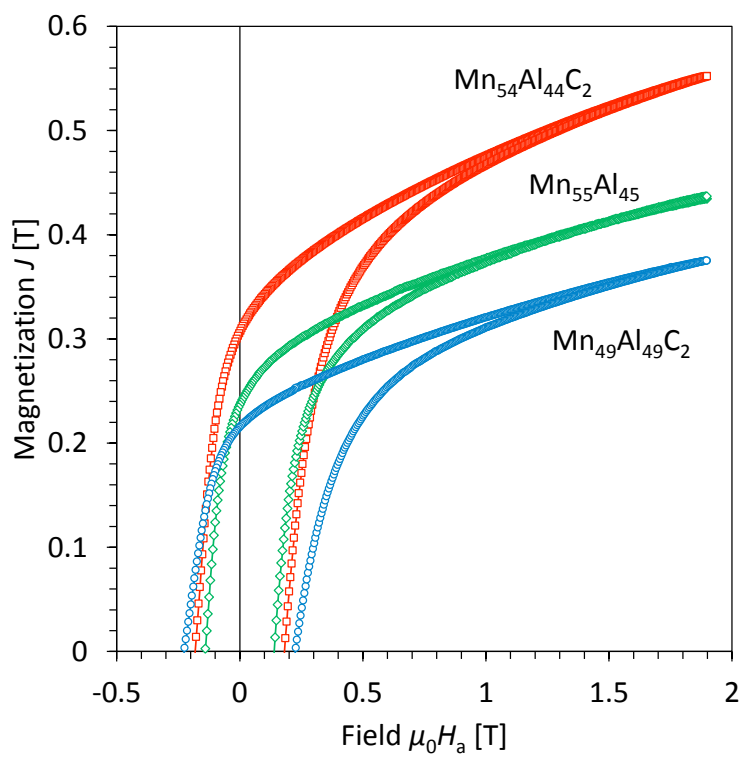

Fig. 3. Magnetization curves of the samples annealed at $823 \mathrm{~K}$.

Figure 3 presents the room temperature magnetization curves of three samples having ferromagnetic behaviour; other samples showed very low values of magnetization. These results agree well with XRD data (Table 1) as $\tau$ phase responsible for hard magnetism is observed after annealing only in $\mathrm{Mn}_{55} \mathrm{Al}_{45}, \mathrm{Mn}_{54} \mathrm{Al}_{44} \mathrm{C}_{2}$ and $\mathrm{Mn}_{49} \mathrm{Al}_{49} \mathrm{C}_{2}$. Moreover, there is a correlation between the weight fraction of $\tau$-phase determined from XRD and the saturation magnetization extracted from VSM measurements. Single-phase alloy $\mathrm{Mn}_{54} \mathrm{Al}_{44} \mathrm{C}_{2}$ has the highest magnetization and better coercivity of $0.19 \mathrm{~T}$ compared to binary alloy $\mathrm{Mn}_{55} \mathrm{Al}_{45}$. Two-phase alloy $\mathrm{Mn}_{49} \mathrm{Al}_{49} \mathrm{C}_{2}$ displays lower magnetization and higher coercivity of $0.23 \mathrm{~T}$. Our coercivity values are higher than $0.17 \mathrm{~T}$ [6] and $0.15 \mathrm{~T}$ [7] reported for other rapid solidification attempts, but lower compared to $0.48 \mathrm{~T}$ [10] achieved in a ball milled powder. However, pure $\tau$-phase can be difficult to obtain in mechanically activated nanostructured material [10].

Figure 4 shows the magnetization of as-quenched $\mathrm{Mn}_{54} \mathrm{Al}_{44} \mathrm{C}_{2}$ sample as a function of temperature under a constant magnetic field of $1.4 \mathrm{~T}$. The ramps were performed between $300 \mathrm{~K}$ and $900 \mathrm{~K}$ at $5 \mathrm{~K} / \mathrm{min}$ rate. Precursor $\varepsilon$-phase is antiferromagnetic below $97 \mathrm{~K}$ [17] and paramagnetic at initial room temperature. On heating, $\varepsilon$-phase transforms to $\tau$-phase which is also paramagnetic at the transition temperature $\sim 786 \mathrm{~K}$ (see the inset). The structural transition is detected due to a difference in magnetic susceptibility of the phases. On cooling, magnetization is increased drastically below Curie temperature of $\tau$-phase as the reverse transition to $\varepsilon$-phase does not occur, probably, due to kinetic reasons.

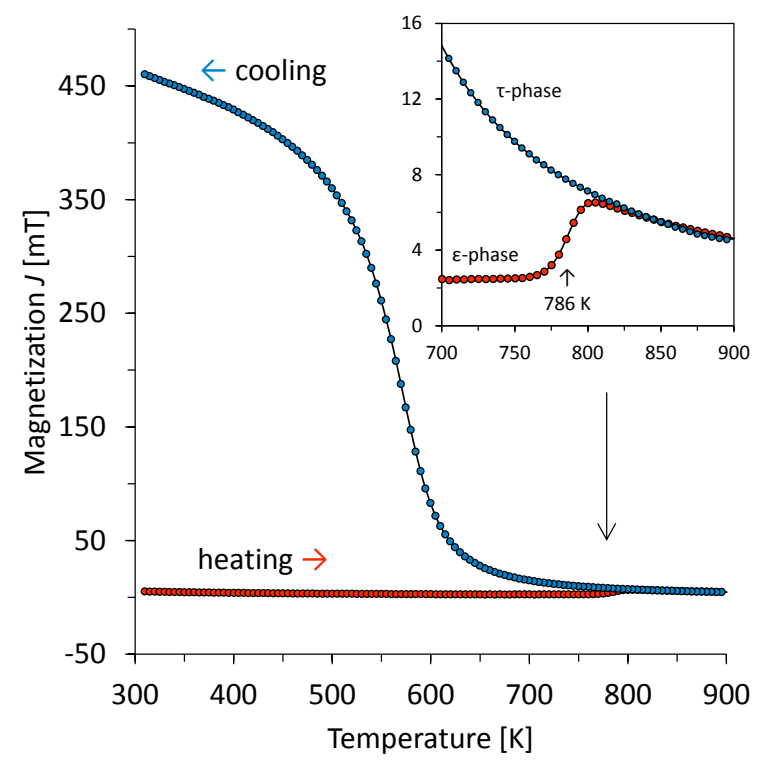

Fig. 4. Magnetization as a function of temperature under applied magnetic field for as-quenched $\mathrm{Mn}_{54} \mathrm{Al}_{44} \mathrm{C}_{2}$ sample. Inset: a magnified fragment of the curve showing the temperature induced $\varepsilon \rightarrow \tau$ structural phase transition.

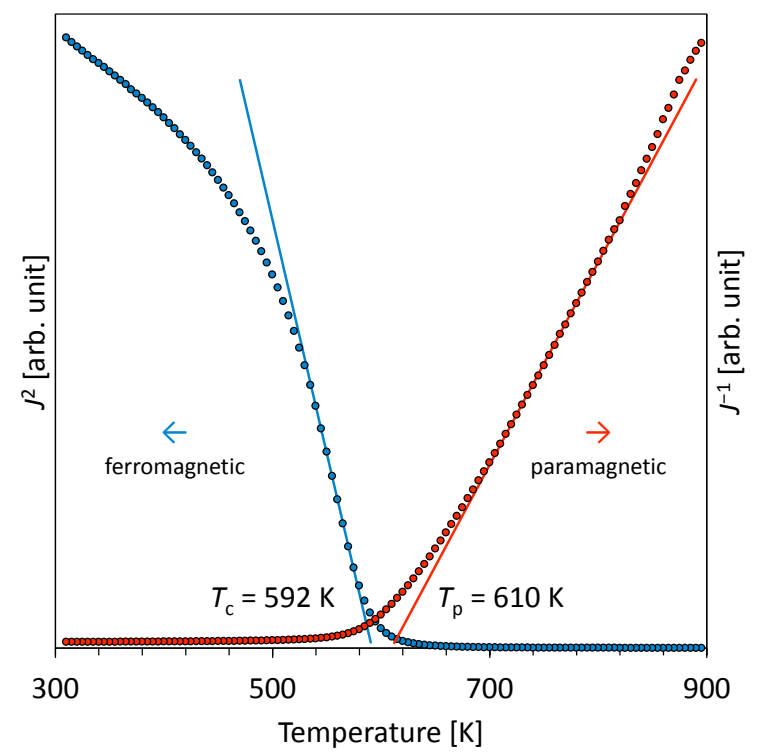

Fig. 5. Determination of Curie temperature from the magnetization measurements in ferromagnetic and paramagnetic states of $\tau$-phase.

Figure 5 illustrates the precise determination of Curie temperature by fitting the inverse magnetization in the paramagnetic state $\left(T_{\mathrm{p}} \approx 610 \mathrm{~K}\right)$ and the square of magnetization in the ferromagnetic state $\left(T_{\mathrm{c}} \approx 592 \mathrm{~K}\right)$ with linear temperature functions. The approximations 
used follow from the temperature dependence of magnetic susceptibility in the mean field theory: $\left(T-T_{\mathrm{p}}\right)^{-1}$ in paramagnetic region and $\left(T_{\mathrm{c}}-T\right)^{1 / 2}$ in ferromagnetic region. Calculated temperatures $T_{\mathrm{p}}$ and $T_{\mathrm{c}}$ correspond well, satisfying usual inequality $T_{\mathrm{p}}>T_{\mathrm{c}}$. Thus, the variable temperature VSM experiment allows to observe $\varepsilon \rightarrow \tau$ phase transition, receive $\tau$-phase (under magnetic field) for further investigation and measure its Curie temperature by two methods.

In Mn-Al-C alloys Curie temperature of $\tau$-phase decreases with carbon doping, available data largely vary $[6,7,10]$. Taking into account this dependence, we find good agreement of our $T_{\mathrm{c}}$ measurements with results of Zeng et al. [10]. The coercivity of $\mathrm{Mn}_{54} \mathrm{Al}_{44} \mathrm{C}_{2}$ sample subjected to in situ annealing is $0.19 \mathrm{~T}$, i.e. equals to that after furnace annealing, and increases to $0.23 \mathrm{~T}$ on cooling down to $100 \mathrm{~K}$.

\section{Conclusions}

Melt-spinning technique enabled us to produce homogeneous single-phase precursors for Mn-Al-C permanent magnets, though higher cooling rate is desirable for reducing the grain size. The metastable ferromagnetic $\tau$-phase has been obtained by annealing non-magnetic $\varepsilon$-phase samples either in a vacuum furnace at $823 \mathrm{~K}$ for $10 \mathrm{~min}$ or directly in the vibrating sample magnetometer under applied magnetic field. Among the studied alloys, best magnetic properties are demonstrated by $\mathrm{Mn}_{54} \mathrm{Al}_{44} \mathrm{C}_{2}$ samples due to the positive effect of carbon on the stability of $\tau$-phase and domain wall pinning by fine carbide precipitates. The coercivity decreases with temperature from $0.23 \mathrm{~T}$ at $100 \mathrm{~K}$ to $0.19 \mathrm{~T}$ at $300 \mathrm{~K}$ and is independent on the annealing method (with or without field). On heating in VSM $\mathrm{Mn}_{54} \mathrm{Al}_{44} \mathrm{C}_{2}$ alloy undergoes the structural $\varepsilon \rightarrow \tau$ phase transformation at $786 \mathrm{~K}$, on cooling Curie temperature of $\tau$-phase $\left(T_{\mathrm{c}}=592 \mathrm{~K}, T_{\mathrm{p}}=610 \mathrm{~K}\right)$ is determined using mean field approximations in ferromagnetic and paramagnetic regions. The results of magnetization measurements are in good agreement with XRD quantitative phase analysis based on Rietveld refinement. The tetragonal distortion of $\tau$-phase unit cell has been confirmed to increase with carbon content, which suggests a possible presence of carbon atoms in the interstitial positions similar to $\mathrm{Fe}-\mathrm{C}$ martensite.

\section{Acknowledgements}

This work was partially supported by ANR project "Micro-MANIP".

\section{References}

1. H. Kono, J. Phys. Soc. Jpn. 13, 1444 (1958).

2. A.J.J. Koch, P. Hokkeling, M.G. v.d. Steeg, K.J. de Vos, J. Appl. Phys. 31, 75S (1960).

3. M.A. Bohlmann, J.C. Koo, J.H. Wise, J. Appl. Phys. 52, 2542 (1981).
4. J.M.K. Wiezorek, A.K. Kulovits, C. Yanar, W.A. Soffa, Metall. Mater. Trans. A 42, 594 (2011).

5. Y. Sakka, M. Nakamura, K. Hoshimoto, J. Mater. Sci. 24, 4331 (1989).

6. E. Fazakas, L.K. Varga, F. Mazaleyrat, J. Alloy. Compd. 434-435, 611 (2007).

7. Z.W. Liu, C. Chen, Z.G. Zheng, B.H. Tan, R.V. Ramanujan, J. Mater. Sci. 47, 2333 (2012).

8. Y.J. Kim, J.H. Perepezko, J. Appl. Phys. 71, 676 (1992).

9. A.E. Berkowitz, J.D. Livingston, J.L. Walter, J. Appl. Phys. 55, 2106 (1984).

10. Q. Zeng, I. Baker, J.B. Cui, Z.C. Yan, J. Magn. Magn. Mater. 308, 214 (2007).

11. J.-G. Lee, X.-L. Wang, Z.-D. Zhang, C.-J. Choi, Thin Solid Films 519, 8312 (2011).

12. T. Saito, J. Appl. Phys. 97, 10F304 (2005).

13. L. Lutterotti, Nucl. Instrum. Methods Phys. Res. B 268, 334 (2010).

14. A. Shukla, A.D. Pelton, J. Phase Equilib. Diffus. 30, 28 (2009).

15. J.-L. Yang, H.-M. Zhou, J.-X. Zhu, X.-X. Zeng, B.S. Zhang, L. Jin, J. Appl. Phys. 55, 2053 (1984).

16. C.T. Lee, K.H. Han, I.H. Kook, W.K. Choo, J. Mater. Res. 7, 1690 (1992).

17. J.J. Wysłocki, P. Pavlik, A. Przybył, Mater. Chem. Phys. 60, 211 (1999). 